\title{
Vorwort zur 2. Auflage
}

Die Kathodenstrahlröhren haben in den letzten Jahren eine stets wachsende Bedeutung erlangt. Als Oszillograph z. B. sind sie zu einem unübertroffenen Hilfsmittel bei allen Schwingungsuntersuchungen geworden, im Fernsehen werden sie als Bildwiedergabegerät im Empfänger und auch zur Bildaufnahme im Sender benutzt und viele Forschungsergebnisse in der modernen Naturwissenschaft sind erst durch exakte Messungen mit solchen Röhren möglich geworden.

Die rasche Verbreitung der Kathodenstrahlröhren in Wissenschaft und Technik bedingt, daß ein wachsender Kreis von Forschern und Technikern mit derartigen Röhren in Berührung kommt. Um mit den Röhren richtig und verständnisvoll arbeiten zu können, muß man aber von der grundsätzlichen Arbeitsweise gewisse Vorstellungen haben.

Das vorliegende Buch will eine solche Einführung in die Grundlagen der Kathodenstrahlröhren geben, in allgemeinverständlicher Darstellung, nur unter Voraussetzung gewisser elektrotechnischer Grundkenntnisșe. Dabei glauben wir, daß auch für den Fachmann das Buch als eine in der vorliegenden Form wohl noch nicht vorhandene Zusammenfassung von Interesse und Wert sein kann.

Die Absicht, im wesentlichen nur die grundsätzlichen Vorgänge klar zu legen, bedeutete einen unvermeidlichen Verzicht auf die Darstellung interessanter theoretischer und spezieller Probleme (wie z. B. die Grenzleistungsfähigkeit der Röhren, Probleme der Hochfrequenzoszillographie, Mikrooszillographie usw.). Andrerseits schien uns aber gerade eine übersichtliche Darstellung der Grundlagen in der Literatur zu fehlen, die dem Leser die notwendigen Grundkenntnisse für das eigene Arbeiten und zum Verständnis der Spezialliteratur vermittelt.

Weil die Grundlagen trotz der mannigfach variierenden Ausführungsformen der Röhren in der Technik die gleichen bleiben, konnten wir die vorliegende zweite Auflage nahezu unverändert gegenüber der ersten (im Jahre 1944 herausgegebenen) Auflage lassen, obwohl nicht alle der in den Abbil- 
dungen gezeigten Röhren z. Zt. im Handel sind, bzw. noch nicht wieder hergestellt oder durch andere Formen in der kommenden Fertigung ersetzt werden. Die Bilder zeigen aber das Typische, und darauf kommt es in diesem Rahmen an.

Die früher benutzten Kathodenstrahlröhren mit kalter Kathode und solche mit Glühkathode und Gasfüllung werden nicht besprochen, da sie nur noch selten Verwendung finden und weil man heute praktisch nur noch Hochvakuumröhren mit Glühkathode baut.

Die praktische Erfahrung lehrt, daß die Erfolge bei dem Arbeiten mit Kathodenstrahlröhren zu einem großen Teil von dem richtigen Zusammenwirken der Röhre mit den angeschlossenen Betriebsgeräten abhängt. Aus diesem Grunde haben wir im letzten Abschnitt auch die Grundlagen dieser Geräte behandelt.

Unser Dank gebührt den verschiedenen Firmen (Telefunken GmbH, A. E. G., Fernseh GmbH, Opta Radio AG, Philips, Siemens \& Halske) für die freundliche Überlassung von Abbildungen der von ihnen hergestellten Röhren und Geräte, und ebenso dem Verlag, der diesen Neudruck trotz der Fülle der nach dem Krieg notwendigen Druckarbeiten so verständnisvoll bevorzugte.

Marburg/Bad Godesberg, August 1950

Die Verfasser 